\section{Arbeit und Ökologie - Gedanken zu einer nachhaltig umweltgerechten Arbeit}

Der große Spannungsbogen von betrieblicher Organisation der Arbeit, der gesellschaftlichen Verteilung von Arbeit und der stofflichen Basis von Arbeit und den damit jeweils verbundenen gesellschaftlichen Regelungen (Recht, Normen etc.pp.) und Wertschätzungen wurde bisher im Zusammenhang mit der Zielorientierung einer nachhaltig umweltgerechten Entwicklung nur am Rande und wenig systematisch behandelt. Mit diesem Artikel möchte ich einen Impuls geben, dies verstärkt zu tun. Dabei konzentriere ich mich angesichts der bereits vorliegenden Beiträge zu diesem Thema auf die stofflich-technischen Aspekte der Arbeit aus Sicht des individuellen Arbeitsvermögens.

Das Thema »Arbeit und Ökologie« ist mir in den letzten drei Monaten aus ganz unterschiedlichen Anlässen begegnet. Erstens habe ich mit anderen Kollegen und Kolleginnen einen Tagungsband über das Verhältnis von Arbeitsschutz und Umweltschutz herausgegeben ${ }^{1}$, zweitens steht bei der. Umsetzung regionaler Entwicklungs- und Standortkonzepte immer wieder die Frage im Mittelpunkt, welche und wieviele Arbeitsplätze denn eine ökologische oder nachhaltige Regionalentwicklung schaffen würde ${ }^{2}$ und drittens habe ich an einem Workshop des Wuppertal-Instituts zum Thema >Arbeitszeit und Ökologie< teilgenommen, auf dem gegen Ende der Veranstaltung von Helmut Spitzley (Universität Bremen) die kurze und doch komplizierte Frage aufgeworfen wurde: »Was hat Arbeit eigentlich mit Ökologie zu tun?«

Das Leitbild der nachhaltigen und umweltgerechten Entwicklung orientiert auf einen haushälterischen Umgang mit natürlichen Ressourcen und Potentialen, wobei unter Natur hier die äußere Natur, die menschliche Umwelt verstanden wird. Der Mensch als Teil der Natur mit seiner biologischen Physis und seiner Einbindung in natürliche Stoffwechselprozesse ist im $\mathrm{Zu}$ sammenhang mit einer nachhaltigen Entwicklung bisher kaum thematisiert. Allein die Formel vom Erhalt der natürlichen Lebensgrundlagen signalisiert, daß der Mensch auch Teil der Natur ist und nicht nur ihr Gegenüber. Um naturalistische Fehlschlüsse zu vermeiden, sollte jedoch beachtet werden, daß der Mensch sich von der Natur auch wesentlich unterscheidet. »In der Natur >geschieht etwas<, der Mensch handelt mit Bewußtsein « ${ }^{3}$.

\section{Soziale und natürliche}

\section{Voraussetzungen der Reproduktion}

Einen quantitativen Zusammenhang von Arbeit und Ökologie sehe ich insbesondere auf folgenden Ebenen:

- Arbeitsprozesse (ökologische Wirkungen von technik, Arbeitsorganisation und eingesetzten Materialien);

- Arbeitsergebnisse (ökologische Wirkungen der Produkte und Dienstleistungen bei der Nutzung, Abfallproblematik);

- Arbeitstätigkeiten und -leistungen (hinsichtlich der sozialen und natürlichen Reproduktionsvoraussetzungen der Arbeitskraft).
Insbesondere der letzte Punkt ist wenig thematisiert und von daher will ich versuchen, den Begriff der sozialen und natürlichen Reproduktionsvoraussetzungen und die damit verbundenen Problemdimensionen thesenartig zu beschreiben:

(1) Ernährung, Schlaf und Ruhe, Regenerationsräume, Erholungsphasen etc. pp. sind die Voraussetzung für jegliche menschliche $\mathrm{Ar}$ beitsleistung. Aus dieser Perspektive ist die Umweltgefährdung gleichzeitig auch eine Zerstörung oder Beeinträchtigung der Regenerationspotentiale der lebendigen Arbeit. Dies ist aber nur ein Aspekt. de, war es aus der Perspektive des gesamten Produktionsprozesses möglich, sich auch von den zeitlichen Regenerationserfordernissen der lebendigen Arbeit unabhängiger zu machen. Dies geschah letztlich auf Kosten der Natur, denn an die Stelle der unmittelbaren Nutzung physischer Kraft trat die. Nutzung natürlicher Energiequellen. Auf der anderen Seite wurde die physiche Energie, die vorher nützlich verausgabt wurde, mehr und mehr in private Freizeitaktivitäten gesteckt. Aus ökologischer Sicht ist hier ein Ungleichgewicht zwischen physischem Leistungsvermögen der Arbeit und der (Über)-Nutzung der natürlichen Potentiale entstanden.

(3) Arbeit war lange Zeit auch immer unmittelbare Bearbeitung von Natur (Landschaft, Rohstoffe, Material). Dieses unmittelbare Verhältnis von Arbeit und Natur wurde durch die moderne Industriearbeit bis auf wenige Ausnahmen aufgelöst. Technik und Arbeitsteilung veränderten die Bèziehung zwischen den Arbeitenden und der Natur grundlegend, sie erleichterten vieles, versperrten jedoch gleichzeitig den Weg für eine sinnliche und ganzheitliche Erfahrung von $\mathrm{Na}-$ tur. Die industrielle Arbeitsorganisation führt zu einer Zersplitterung der Arbeit und damit auch zu einer Segmentierung des Bewußtseins über die Natur. Daß am Ausgangspunkt und am Ende jeder Produktion riesige Stoffwechselleistungen der Natur stehen (und dabei das Leistungsvermögen der Natur durch die derzeitige Produktionweise stetig untergraben wird) ist vielen Kopfarbeitern (und die sind inzwischen in der Mehrzahl) nicht mehr bewußt. Die abstrakte Arbeit hat sich von ihren natürlichen Grundlagen in doppelter Weise entfernt: real und gedanklich.
(2) In dem Maße wie die Arbeit technisiert wur-
(4) Im Zuge dieser Entwicklung hat sich auch die gesellschaftliche Wertschätzung von Arbeit verändert. Generell wird geistige Arbeit mehr geschätzt und höher entlohnt als körperliche $\mathrm{Ar}$ beit. Einfache Tätigkeiten zur Befriedigung von Grundbedürfnissen (z.B. Essen zubereiten, Pflege von Menschen und Natur) werden verhältnismäßig schlecht entlohnt und sind wenig anerkannt. Hinzu kommt, daß in der bundesrepublikanischen Gesellschaft immer weniger ein grundlegendes Wissen und Fähigkeiten zur Reproduktion der Grundbedürfnisbereiche vorhanden sind. Ein generalisierbares, meist handwerkliches Grundwissen wurde früher in den Familien oder anderen sozialen Zusammenhängen weitervermittelt. Hierfür fehlt heute die soziale Instanz bzw. auch die Bereitschaft, in den Bildungseinrichtungen entsprechendes Wissen $\mathrm{zu}$ vermitteln. Erschwerend ist des weiteren, daß es kaum noch Menschen gibt, die diese Qualifikationen und Fertigkeiten noch haben und vermitteln könnten.

(5) Das Normalarbeitsverhältnis ${ }^{4}$ (der erwerbsorientierte Vollzeitberuf von Männern) in der Industriegesellschaft basiert auf den Reproduktionsleistungen von Frauen, die quasi die haushälterische Basis für das Arbeitsvermögen des Mannes darstellen ${ }^{5}$. Die damit einhergehende Rollenzuweisung wird mit Recht kritisiert. Da immer mehr Frauen am Erwerbsleben teilnehmen, die Männer jedoch nicht in gleichem Maße bereit sind, Aufgaben der Haushaltsführung und Reproduktion wahrzunehmen, entsteht eine »Reproduktionslücke«: Es gibt immer mehr Normalarbeitsverhältnisse ohne Normalfamilie oder andere soziale Zusammenhänge als Reproduktionsinstanzen. Infolgedessen wird die individuelle Reproduktion der Normalarbeit zum Gegenstand neuer Dienstleistungsangebote, in denen wiederum in der Mehrzahl Frauen tätig sind. $\mathrm{Ob}$ dies aus arbeitspolitischer und aus emanzipatorischer Sicht eine wünschenswerte Entwicklung ist, muß bezweifelt werden. Das reproduktive Verhältnis von Natur und Arbeit verliert in dem Maße an Qualität, wie die Einbindung in den sozialen Kontext verloren geht.

\section{Haushälterischer Umgang} mit dem Arbeitsvermögen

Ich greife diese `ökologischen « Stränge der $\mathrm{Ar}$ beitsdebatte auch auf, weil in den aktuellen Beiträgen zur $>$ Arbeitswelt der Zukunft $<$ kaum Perspektiven für eine Ökologisierung der Arbeit enthalten sind ${ }^{6}$. Bereits die Problemorientierung ist so angelegt, da $\beta$ im Ergebnis nur über den Wandel der Arbeit, der auf sozialen Tatbeständen beruht, diskutiert wird. Demgegenüber möchte ich deutlich machen: Arbeit ist auch immer in einen Stoffwéchselprozeß mit der Natur eingebunden, und zwar nicht nur über die gegenständliche Seite der Arbeit - ihre Produkte und Dienstleistungen (Outputs) - sondern auch und gerade über die physischen, kognitiven und mentalen Leistungen (Inputs) der Menschen. Das individuelle Arbeitsvermögen hat eine 
natürliche, ökologische Basis und auch diese unterliegt einem dramatischen Wandel. Von daher sollte das Ziel eines haushälterischen Umgangs mit der Natur einen haushälterischen Umgang mit dem Arbeitsvermögen (gesellschaftlich und individuell) einschließen. Es ist die Frage zu stellen, ob das herkömmliche, industrielle Rationalisierungsprogramm ,Energie und Technikeinsatz statt Arbeit < in einer nachhaltigen Wirtschaft begrenzt werden bzw. ob in einigen Teilbereichen der Arbeitswelt (z. B. der Landwirtschaft, Befriedigung der Grundbedürfnisse, Erholung) nicht ein umgekehrter Prozeß (>Arbeit statt Energie $<$ ) einsetzen sollte. ${ }^{7}$ Ein haushälterischer Umgang mit der lebendigen Arbeitskraft ist aus dieser Sicht mit folgenden Anforderungen verbunden:

- Der ökologische Wert physischer Anstrengung im Arbeitsprozess oder bei der Fortbewegung ist anzuerkennen und neu zu bewerten.

- Das Verhältnis von Hand- und Maschinenarbeit entlang eines energieorientierten Optimums ist näher zu bestimmen.

- Eine sozial, ökologisch und kulturell eingebundene Regenerationsbasis der lebendigen Arbeit ist zu sichern.

- Der Stoffwechsel mit der Natur muß in einer nachhaltigen Arbeitsumwelt wieder erfahrbar sein (Rückkopplung der Effekte bezogen auf den Betrieb, den Haushalt, die Kommune/Region).

Alle vier Punkte können in ihrer weiteren Ausgestaltung letzlich zu einer konkreten Utopie beitragen, in der das Verhältnis von Kopf- und Handarbeit und Arbeitsleistung und Reproduktion/Regeneration neu bestimmt wird, mit dem Ziel, die derzeitigen, umweltschädlichen und naturfernen Normalarbeitsverhältnisse zu verändern. In der Konkretisierung einer solchen Utopie gibt es jedoch einige Konfkliktfelder mit anderen $>$ Arbeitsorientierungen $<$. Bezogen auf einige Diskurse über die Zukunft der Arbeit will ich ohne den Anspruch auf Vollständigkeit vier benennen:

- Die engen funktionsorientierten Schutzstrategien, die Arbeit und Umwelt getrennt mit Hilfe von Grenzwerten und restriktiven Schutzbestimmungen (Ordnungsrecht, Arbeitszeitordnung) erhalten wollen, müssen um die Fragestellung eines neuen Verhältnisses von Arbeit und Umwelt und der Konsequenzen hieraus für die Gestaltung der $\mathrm{Ar}$ beitsprozesse (technisch-instrumenteller Anteil, welche Technik) erweitert werden.

- Ansätze für eine neue Arbeitszeitpolitik ${ }^{8}$ dürfen nicht bei den Fragen der Verteilungsgerechtigkeit (Entlohnung, Alterssicherung) und der Geschlechterrollenproblematik stehen bleiben, sondern müssen sich auch den Fragen nach dem (individuellen und gesellschaftlichen) Sinn von Arbeit und Freizeit stellen. Diese Sinnfragen sind angesichts ausdifferenzierter und vielfältiger Lebensformen und Milieus sicher nicht mit einem einheit- lichen »Wohlstandsmodell« zu lösen. Als Perspektive sehe ich hier eher einen Flickenteppich von vielen Arbeitskulturen und vielen Konsumkulturen, die sich vielleicht über einen Modus des Nebeneinander verständigen müssen, ohne die anderen zu zerstören und zu verdrängen (Toleranzprinzip). ${ }^{9}$

- Ansätze zu einer Modernisierung alter Industrieregionen ${ }^{10}$ sollten nicht das Problem umgehen, ob die arbeitenden Menschen subjektiv bereit und objektiv in der Lage sind, in den vom Wettbewerb diktierten Modernisierungswellen mitzuschwimmen (vom Surfen auf den Wellen ganz zu schweigen). Das Verhältnis von traditioneller Arbeit und moderner Arbeit, von einfacher und hochtechnisierter Arbeit muß anhand des tatsächlichen, regionalen Arbeitsvermögens neu bestimmt werden. Insofern brauchen die Regionen/ regionale Arbeitsmärkte auch Bewahrungsstrategien: Produktionskulturen und Arbeitserfahrungen zur Befriedigung der Grundbedürfnisse (Ernähren, Bauen, Kleiden, Erholung) sollten regional eingebunden bleiben

1) vgl. Hildebrandt, E./ Linne, G./ Lucas, R./ Sieben, G. (Hrsg.): Arbeitsschutz und Umweltschutz - zwischen Eigenständigkeit und Gemeinsamkeit, Düsseldorf 1994 (Graue Reihe der Hans Böckler Stiftung).

2) Vor dem Hintergrund des drohenden Verlustes vieler verwaltungsorientierter Arbeitsplätze in Bonn wurde das IÖW von der Hans Böckler Stiftung beauftragt, die Potentiale und Perspektiven ökologischer Dienstleistungen zu untersuchen

3) v. Gleich, A./Lucas, R./Schleicher, R./Ullrich, O.: Blickwende in der Technologiepolitik, Opladen 1992, S. 15

4) Mit dem Begriff des Normalarbeitsverhältnisses werden die individuellen und gesellschaftlichen Handlungsstrukturen gekennzeichnet, die weitgehend durch Recht und Normen verfestigt sind (z.B. Arbeitszeitregelungen, Entlohnungsformen, soziale Sicherung und geschlechtsspezifische Arbeitsteilung)

5) vgl. hierzu ausführlich: Eckart, C.: Die verleugneten Voraussetzungen des >Normalarbeitsverhältnisses< und ihre Wiederkehr in weiblichen Berufsbiographien, Kassel 1994

\section{Adressen}

\section{IÖW/VÖW-Geschäftsstelle}

Giesebrechtstraße 13, 10629 Berlin

Tel: (030) 884 5940, Telefax 8825439

ACHTUNG: NEUE TELEFONNUMMER!

\section{IÖW-Regionalbüro}

Nordrhein-Westfalen

Völklinger Straße 9, 42285 Wuppertal

Tel. (0202) 80530, Telefax 83402 und durch politische Maßnahmen geschützt und gestützt werden.

- Dualwirtschaftliche Strategien ${ }^{11}$, die auf eine Veränderung der Erwerbsarbeit und der Eigenarbeit zielen, sollten beachten, daß die Eigenarbeit und Hausarbeit sich nicht zu einem Anhängsel einer modernisierten Erwerbsarbeit entwickeln, dem die Aufgaben des Ausgleichs und der Reproduktion/ Regeneration zugewiesen werden.

Aus einer derartigen ökologischen Erweiterung der Arbeit und deren Verbindung mit sozialen Entwicklungen können sich auch neue Perspektiven für das Verhältnis von Arbeiten und Leben eröffnen. Nachhaltiges Arbeiten braucht soziale und regionale Bindung als Bestandteil einer Kultur, die sich insgesamt neu entwickeln muß. Ob die Arbeit dabei wieder mehr als heute der Dreh- und Angelpunkt der gesellschaftlichen Entwicklung sein sollte oder auch nicht, ist eine spannende Frage, die noch zu beantworten wäre.

Rainer Lucas, Wuppertal

6) vgl. z.B. Mathies, H./Mückenberger, U./ Offe, C./Peter, E./ Raasch, S.: Arbeit $2000-$ Anforderungen an eine Neugestaltung der Arbeitswelt, Reinbek 1994 oder auch: Klauder, W.: Ohne Fleiß kein Preis - Die Arbeitswelt der Zukunft, Zürich/Osnabrück 1990. In letzterer wird zumindest der klassische Umweltschutz thematisiert.

7) vgl. hierzu auch Bode, W.: Die Landschaft als Arbeitsplatz zurückgewinnen! - Der Grüne Arbeitsmarkt - ein Modell für die Zukunft, in:Land nutzen - Natur schützen - von der Konfrontation zur Kooperation, hrsg. v. Evangelische Akademie Baden, Karlsruhe 1995

8) Garhammer, M.: Balanceakt Zeit - Auswirkungen flexibler Arbeitszeiten auf Alltag, Freizeit und Familie, Berlin 1994

9) vgl. hierzu auch Bierter, W./ v. Winterfeld, U. : Jenseits von Arbeit und Konsum?, in: Politische Ökologie Nr. 23, 11. Jg., S. 20-23

10) vgl, z. B. Kilper/Latniak/Rehfeld/Simonis: Das Ruhrgebiet im Umbruch, Opladen 1994

11) vgl. z. B. Huber, J.: Die zwei Gesichter der Arbeit. Ungenutzte Möglichkeiten der Dualwirtschaft. Berlin 1983

\section{IÖW-Regionalbüro}

\section{Baden-Württemberg}

Bergheimerstraße 97,69115 Heidelberg

Tel. (06221) 167954, Telefax 27060

\section{IÖW-Projektbüro}

Hannover

Hausmannstraße 9, 30159 Hannover

Tel. (05 11) 1640344, Telefax 1640391 
(c) 20I0 Authors; licensee IÖW and oekom verlag. This is an article distributed under the terms of the Creative Commons Attribution Non-Commercial No Derivates License (http://creativecommons.org/licenses/by-nc-nd/3.o/), which permits unrestricted use, distribution, and reproduction in any medium, provided the original work is properly cited. 